Published in final edited form as:

Pediatr Transplant. 2013 May ; 17(3): E104-E107. doi:10.1111/petr.12068.

\title{
Successful Autologous Cord Blood Transplantation in a Child with Acquired Severe Aplastic Anemia
}

\author{
David Buchbinder, MD MS ${ }^{\star *}$, \\ Division of Hematology CHOC Children's Hospital 455 S. Main Street Orange, CA 92868 \\ Loah Hsieh, MD, \\ Division of Hematology CHOC Children's Hospital 455 S. Main Street Orange, CA 92868 Work \\ phone 714 / 532 - 8459 lhsieh@choc.org \\ Geetha Puthenveetil, MD, \\ Division of Hematology CHOC Children's Hospital 455 S. Main Street Orange, CA 92868 Work \\ phone 714 / 532 - 8459 Fax 714 / 532 - 8771 gputhenveetil@choc.org
}

\author{
Amit Soni, MD, \\ Division of Hematology CHOC Children's Hospital 455 S. Main Street Orange, CA 92868 Work \\ phone 714 / 532 - 8459 asoni@choc.org \\ Jill Stites, FNP, \\ Division of Hematology CHOC Children's Hospital 455 S. Main Street Orange, CA 92868 Work \\ phone 714 / 532 - 8459 jstites@choc.org

\section{Van Huynh, MD,} \\ Division of Oncology CHOC Children's Hospital 455 S. Main Street Orange, CA 92868 Work \\ phone 714 / 532 - 3636 vhuynh@choc.org \\ Ivan Kirov, MD, \\ Division of Oncology CHOC Children's Hospital 455 S. Main Street Orange, CA 92868 Work \\ phone 714 / 532 - 3636 ikirov@choc.org
}

\section{Steve Neudorf, MD,}

Division of Oncology CHOC Children's Hospital 455 S. Main Street Orange, CA 92868 Work phone 714 / 532-3636 sneudorf@choc.org

\section{Elyssa Rubin, MD,}

Division of Oncology CHOC Children's Hospital 455 S. Main Street Orange, CA 928868 Work phone 714 / 532-3636 erubin@choc.org

\section{Leonard Sender, MD,}

Division of Oncology CHOC Children's Hospital 455 S. Main Street Orange, CA 92868 Work phone 714 / 532-3636 Isender@choc.org

\section{Lilibeth Torno, MD,}

Division of Oncology CHOC Children's Hospital 455 S. Main Street Orange, CA 92868 Work phone 714 / 532-3636 Itorno@choc.org

\footnotetext{
** Denotes the corresponding author Work phone 714 / 532 - 8459 Fax 714 / 532 - 8771 dbuchbinder@ choc.org.
} 
David Margolis, MD,

Bone Marrow Transplantation Children's Hospital of Wisconsin 8701 Watertown Plank Road

Wauwatosa, WI 53226 Work phone 414 / 456-4154 dmargoli@mcw.edu

Richard Childs, MD,

Transplantation Immunotherapy National Institutes of Health 31 Center Drive MSC 2486

Bethesda, MD 20892 Work phone 301 / 451-7128 childsr@nhlbi.nih.gov

Theodore Moore, MD, and

Division of Oncology Mattel Children's Hospital at UCLA 10833 Le Conte Avenue Los Angeles, Ca 90095 Work phone 310 / 825 - 6708 tbmoore@mednet.ucla.edu

Diane Nugent, MD

Division of Hematology CHOC Children's Hospital 455 S. Main Street Orange, CA 92868 Work phone 714 / 532 - 8459 dnugent@choc.org

\section{Abstract}

Over 400 cases of pediatric severe aplastic anemia (SAA) occur annually in the United States. A growing number of children with SAA may have had their stem cells harvested through cord blood collection. We describe a 9-year-old male with SAA treated successfully with an autologous cord blood transplant following immunoablative chemotherapy. With the increasing number of people cryopreserving autologous cord blood the use of autologous cord blood in the treatment of SAA might be considered as initial therapy. This case serves to discuss approaches to preparative therapy as well as the potential complications in this growing cohort of patients.

\section{Keywords}

Acquired aplastic anemia; cord blood; autologous transplantation

\section{INTRODUCTION}

Hematopoietic cell transplantation (HCT) has been used successfully to treat acquired severe aplastic anemia (SAA) for several decades [1-5]. In the last two decades, a significant improvement in outcomes has been reported in patients with acquired SAA undergoing matched sibling donor or unrelated donor HCT [1-5]. Many patients lack an appropriate donor requiring the use of immunosuppressive therapy and in rare cases other alternative donor HCT approaches including haploidentical and cord blood HCT [6-8]. Autologous HCT for aplastic anemia utilizing peripheral blood stem cells and cord blood has been reported only rarely [9-13]. Three pediatric HCT cases document the successful use of autologous cord blood HCT in pediatric patients with acquired SAA following a preparative regimen of anti-thymocyte globulin. This approach may not provide effective immune ablation and has potential toxicities including infectious complications and systemic toxicities. We describe a 9-year-old Hispanic male diagnosed with acquired SAA who underwent the first successful autologous cord blood transplant following an immunoablative preparative regimen consisting of fludarabine in combination with cyclophosphamide. 


\section{Clinical History}

A previously healthy 9-year-old Hispanic male was referred to our center for evaluation of epistaxis. The past medical history was unremarkable, including a normal delivery and early childhood development. The family history was non-contributory and there was no consanguinity for three generations reviewed. He was noted to have a normal physical examination apart from petechiae and ecchymoses. Laboratory examination revealed a white blood cell count of 2,200 cells / uL (normal 3.5-10.9), hemoglobin of $8.0 \mathrm{~g} / \mathrm{dL}$ (normal 10.9-14.4), hematocrit 21.9\% (normal 32.5-41.5), and platelet count of $<2,000 / \mathrm{uL}$ (normal 150,000-450,000). The mean corpuscular volume was $81.1 \mathrm{fL}$ (normal 76-91) and the absolute reticulocyte count was 17,000 / uL (normal 15,000-105,000). The differential demonstrated 31\% neutrophils (normal 35-75), 51\% lymphocytes (normal 22-58), 13\% monocytes (normal 0-8), $2 \%$ eosinophils (normal 0-5), and 3\% band neutrophils (normal $0-9$ ) with an absolute neutrophil count of 748 cells / $\mathrm{uL}$. A bone marrow examination showed a hypocellular bone marrow with a cellularity of less than 5\% composed of fatty tissue with scattered lymphocytes and stromal elements. Cytogenetic evaluation demonstrated a normal male karyotype. Evaluation for causes of SAA including infectious causes, clonal disorders, paroxysmal nocturnal hemoglobinuria, and inherited bone marrow failure syndromes including Fanconi anemia, dyskeratosis congenita were all negative.

The diagnosis of acquired SAA was made and due to an ongoing requirement for transfusion support, and the lack of a sibling donor, immunosuppressive therapy consisting of equine anti-thymocyte globulin in combination with cyclosporine was initiated. Six months following the start of immunosuppressive therapy, the patient still had no marrow recovery as documented by a need for daily granulocyte colony stimulating factor as well as weekly packed red blood cell and platelet transfusions. An unrelated bone marrow donor search revealed a 9 out of 10 matched unrelated bone marrow donor (HLA A antigen mismatch). Surprisingly, one 6 out of 6 matched cord blood unit was found to be available in the public cord blood registry and further inquiry revealed that this donated cord blood unit was indeed, an autologous unit. Given the lack of response to immunosuppressive therapy, the risks associated with the use of a single antigen mismatched unrelated donor HCT, and the availability of the patient's own cord blood unit, a decision was made to proceed with autologous cord blood HCT. Following discussions regarding the risks, benefits, and alternatives, informed consent was obtained.

The HCT preparative regimen included: fludarabine $30 \mathrm{mg} / \mathrm{m} 2$ / day from Day -6 to Day -4 for a total dose of $90 \mathrm{mg} / \mathrm{m} 2$. Cyclophosphamide $60 \mathrm{mg} / \mathrm{kg} /$ day was given from Day -3 to Day -2 for a total dose of $120 \mathrm{mg} / \mathrm{kg}$ together with MESNA prophylaxis. This was followed by a day of rest on Day -1 and an infusion of $3.0 \times 10 \mathrm{e}^{7} \mathrm{TNC} / \mathrm{kg}$ and $0.9 \times 10 \mathrm{e}^{6}$ $\mathrm{CD} 34+/ \mathrm{kg}$ using an autologous cord blood unit on Day 0. Prophylaxis against sinusoidal obstruction syndrome was achieved using ursodiol at a dose of $6 \mathrm{mg} / \mathrm{kg} /$ dose orally twice a day. Prophylaxis against Pneumocystis jiroveci was provided using aerosolized pentamidine at a dose of $300 \mathrm{mg} /$ dose monthly. Antifungal prophylaxis was provided with micafungin at a dose of $4 \mathrm{mg} / \mathrm{kg} /$ day intravenously. Intravenous immunoglobulin was also provided at a dose of $0.5 \mathrm{~g} / \mathrm{kg} /$ dose and was administered prior to the start of conditioning. 
His transplant course was complicated by a self-limited period of febrile illness and Streptococcus mitis bacteremia requiring antibiotic therapy. On Day +10 , the patient developed radiographic evidence of bilateral pleural effusions and pulmonary edema which resolved. A chest CT demonstrated bibasilar infiltrates, so a bronchoaveolar lavage was performed. All diagnostic fungal, bacterial stains and cultures from these studies were negative. His absolute neutrophil count exceeded 500 cell / uL on Day +27 . His platelet count was greater than $20,000 / \mathrm{uL}$ on Day +36 with his last platelet and packed red blood cell transfusion given on Day +20 . The patient was discharged at D+27 and remains transfusion independent at the time of his most recent clinical assessment at D+100. His Lansky performance status was 100 at discharge and has remained so now at over 3 months post-HCT.

\section{DISCUSSION}

For patients with acquired SAA who fail initial immunosuppressive therapy and lack a well matched unrelated donor, therapeutic options are limited [1-6]. Alternative unrelated donor HCT is a potential option; however, significant risks, including graft-versus-host disease and graft failure remain for those lacking a 10/10 HLA matched unrelated donor [14].

Autologous HCT utilizing peripheral blood stem cells or cord blood provides a clinical approach free of graft-versus-host disease; however, these approaches are subject to significant limitations including efficacy and availability; respectively [9-13, 15]. The case presented in this report suggests that autologous cord HCT may play a more important role in the treatment of patients with acquired SAA.

To our knowledge, this report is the first description of a successful application of autologous cord blood HCT in a pediatric patient with acquired SAA using an immunoablative preparative regimen consisting of fludarabine and cyclosphophamide. Autologous HCT using peripheral blood stem cells has been reported in the treatment of patients with acquired SAA [9-13]. Three successful cases have been reported in adult patients utilizing high dose cyclophosphamide $(200 \mathrm{mg} / \mathrm{kg})$ as a preparative regimen [9-11]. Autologous cord blood HCT has been utilized in four pediatric cases of SAA, with variable success following a preparative regimen of anti-thymocyte globulin [12-13]. Since our patient did not respond to equine anti-thymocyte globulin, a decision was made to explore a chemotherapy-based preparative regimen that would be immunoablative. Based on recent reports of excessive organ toxicity with higher doses of cyclophosphamide, we chose a lower dose of cyclophosphamide $(120 \mathrm{mg} / \mathrm{kg})$ in combination with the lymphoablative agent fludarabine [16]. Post-HCT immunosuppressive with cyclosporine was not utilized.

Autologous cord blood HCT in the setting of acquired SAA is not without risks. More intense chemotherapy will put the patient at risk for infection, but in patients with no myeloid cells, the short-term depletion, may be offset by more rapid engraftment, shortening the duration of neutropenia. The inability to eradicate the ongoing effector cells that mediate associated destruction of hematopoietic progenitor cells may contribute to the risk of recurrence [6]. Other risks also remain including toxicity associated with the preparative regimen. Despite these risks, ongoing research continues to explore the use of autologous HCT for the treatment of other autoimmune diseases [17]. 
When confronting the decision to proceed with unrelated donor HCT the risk to benefit ratio of this approach merits a much more cautious approach. The use of modern preparative regimens for patients with acquired SAA is associated with decreased transplant-related morbidity and mortality. Preparative regimens utilizing decreased or no doses of total body irradiation, decreased doses of cyclophosphamide, and the use of agents including fludarabine and alemtuzumab show promising results for patients with 10/10 matched unrelated donors [1-5]. Faced with the option of pursuing an unrelated donor HCT with a 7 out of 8 allele matched bone marrow donor, we felt that the decision to consider autologous cord blood HCT, which has no risk of graft-versus-host disease, was a reasonable approach for this child who had his own cord blood unit available. This approach was further supported by the excellent outcomes that have been reported in the setting of syngeneic HCT for acquired SAA which shares some clinical similarities [18].

We cannot definitively exclude that the immunoablative properties of the preparative combination of fludarabine and cyclophosphamide were responsible for autologous reconstitution of hematopoiesis in our patient. In addition to the use of anti-thymocyte globulin and cyclosporine, which have been applied successfully in the treatment of acquired SAA, previous data has documented the efficacy of high dose cyclophosphamide without stem cell rescue [19]. Even if this were the case, the kinetics of our patient's response suggests that the cord blood unit engrafted or at least helped to hasten his engraftment and transfusion independence. Lastly, the follow-up is short and the long-term efficacy of this approach is not known.

In summary, these findings suggest that for the patient with a diagnosis of acquired SAA who has failed immunosuppressive therapy, autologous cord blood HCT should be considered if available before considering unrelated donor HCT. For patients with an inherited bone marrow failure syndrome this strategy may be more likely to fail as the quality and / or number of hematopoietic stem cells in the cord blood unit may be poor. We found autologous HCT following an immunoablative combination of fludarabine and cyclophosphamide to be an effective and safe alternative to the use of other alternative donor HCT approaches. Despite the rarity of this scenario, clinical efforts focused on the expansion of a national cord blood collection program and research efforts directed at the generation of hematopoietic stem cells from nonhematopoietic tissue may increase the possibility of using autologous stem cell sources for our growing and increasing diverse population of patients needing HCT [20].

\section{REFERENCES}

1. Kahl C, Leisenring W, Deeg HJ, et al. Cyclophosphamide and antithymocyte globulin as a conditioning regimen for allogeneic marrow transplantation in patients with aplastic anaemia: a long-term follow-up. Br J Haematol. 2005; 130:747-751. [PubMed: 16115132]

2. Deeg HJ, O'Donnell M, Tolar J, et al. Optimization of conditioning for marrow transplantation from unrelated donors for patients with aplastic anemia after failure of immunosuppressive therapy. Blood. 2006; 108:1485-1491. [PubMed: 16684959]

3. Bacigalupo A, Socie G, Lanino E, et al. Fludarabine, cyclophosphamide, antithymocyte globulin, with or without low dose total body irradiation, for alternative donor transplants, in acquired severe aplastic anemia: a retrospective study from the EBMT-SAA Working Party. Haematologica. 95:976-982. [PubMed: 20494932] 
4. Marsh JC, Gupta V, Lim Z, et al. Alemtuzumab with fludarabine and cyclophosphamide reduces chronic graft-versus-host disease after allogeneic stem cell transplantation for acquired aplastic anemia. Blood. 2011; 118:2351-7. [PubMed: 21518925]

5. Samarasinghe S, Steward C, Hiwarker P, et al. Excellent outcome of matched unrelated donor transplantation in paediatric aplastic anaemia following failure with immunosuppressive therapy: a United Kingdom multicentre retrospective experience. Br J Haematol. 2012; 157:339-46. [PubMed: 22372373]

6. Scheinberg P, Young NS. How I treat acquired aplastic anemia. Blood. 2012; 120:1185-96. [PubMed: 22517900]

7. Zhou F, Ge L, Yu Z, et al. Clinical observations on intensive immunosuppressive therapy combined with umbilical cord blood support for the treatment of severe aplastic anemia. J Hematol Oncol. 2011; 4:27. [PubMed: 21663651]

8. Liu HL, Sun ZM, Geng LW, et al. Unrelated cord blood transplantation for newly diagnosed patients with severe acquired aplastic anemia using a reduced-intensity conditioning: high graft rejection, but good survival. Bone Marrow Transplantation. 2012 [Epub ahead of print].

9. Koza V, Jindra P, Svojgrova M, et al. Successful autologous transplantation in a patient with severe aplastic anemia (SAA). Bone Marrow Transplantation. 1998; 21:957-9. [PubMed: 9613793]

10. Balint B, Stamatovic D, Todorovic M, et al. Autologous transplant in the treatment of severe aplastic anemia - A case report. Transfusion and Apheresis Science. 2011; 45:137-141. [PubMed: 21871837]

11. Frangoul H, Navarro WH, Confer DL, et al. Infusion of autologous peripheral blood stem cells in an unrelated donor who developed severe aplastic anemia following stem cell donation. Bone Marrow Transplantation. 2012; 47:869. [PubMed: 21874062]

12. Fruchtman SM, Hurlet A, Dracker R, et al. The Successful Treatment of Severe Aplastic Aenmia with Autologous Cord Blood Transplantation. Biology Blood Marrow Transplantation. 2004; 10:741-42.

13. Rosenthal J, Woolfrey AE, Pawlowska A, et al. Hematopoietic Cell Transplantation With Autologous Cord Blood in Patients With Severe Aplastic Anemia: An Opportunity to Revisit the Controversy Regarding Cord Blood Banking for Private Use. Pediatric Blood Cancer. 2011; 56:1009-1012. [PubMed: 21370429]

14. Yagasaki H, Kojima S, Yabe H, et al. Acceptable HLA-mismatching in unrelated donor bone marrow transplantation for patients with acquired severe aplastic anemia. Blood. 2011; 118:318690. [PubMed: 21757619]

15. Sloand EM, Read EJ, Scheinberg P, et al. Mobilization, collection, and immunomagnetic selection of peripheral blood CD34 cells in recovered aplastic anemia patients. Transfusion. 2007; 47:125053. [PubMed: 17581160]

16. Tolar J, Deeg HJ, Arai S, et al. Fludarabine-based conditioning for marrow transplantation from unrelated donors in severe aplastic anemia: early results of a cyclophosphamide dose deescalation study show life-threatening adverse events at predefined cyclophosphamide dose levels. Biol Blood Marrow Transplant. 2012; 18:1007-11. [PubMed: 22546497]

17. Passweg J, Tyndall A. Autologous stem cell transplantation in autoimmune diseases. Semin Hematol. 2007; 44:278-85. [PubMed: 17961728]

18. Lu DP. Syngeneic bone marrow transplantation for the treatment of aplastic anemia: report of a case and review of the literature. Exp Hematol. 1981; 9:257-63. [PubMed: 7014237]

19. Brodsky RA, Chen AR, Dorr D, et al. High-dose cyclophosphamide for severe aplastic anemia: long-term follow-up. Blood. 2010; 115:2136-41. [PubMed: 20018919]

20. Lesinski DA, Heinz N, Pilat-Carotta S, et al. Serum- and stromal cell-free hypoxic generation of embryonic stem cell-derived hematopoietic cells in vitro, capable of multilineage repopulation of immunocompetent mice. Stem Cells Transl Med. 2012; 1:581-91. [PubMed: 23197864] 